\title{
SUPEROVULAÇÃO OVARIANA E PRODUÇÃO DE EMBRIÕES EM Bubalus bubalis
}

\author{
(Superovulation and embryo production in Bubalus bubalis)
}

\author{
LIPINSKI, L.C.'; KOZICKI, L.E. ${ }^{2}$; MARTINEZ, J.L. ${ }^{3}$; \\ PIASECKI JUNIOR, S.J. ${ }^{4}$; SEGUI, M.S. ${ }^{5}$; WEISS, R.R. ${ }^{6}$
}

'Bolsista PIBIC - PUCPR e Médico Veterinário autônomo; ${ }^{2}$ Teriogenologia PUCPR e UFPR; ${ }^{3}$ IAPAR - Curitiba; ${ }^{4}$ Curso de Medicina Veterinária PUCPR; ${ }^{5}$ Teriogenologia PUCPR; ${ }^{6}$ Teriogenologia UFPR.

\begin{abstract}
RESUMO - O presente estudo objetivou otimizar a superovulação ovariana, a taxa de ovulação e a produção de embriões em búfalas. Os animais eram da raça Murrah e Mediterrâneo e estavam em torno do $60^{\circ}$ dia pós parto. Quatorze animais foram divididos em dois grupos, sendo um grupo tratado ( $G 1=7$ animais) e outro não-tratado (G2=7 animais). Todos os animais foram submetidos à sincronização do estro, recebendo no dia 0 (D0) um pessário vaginal com progestágeno (CIDR-B, Intervet); no dia 1 (D1) pela manhã foi aplicado $3 \mathrm{mg}$ de benzoato de estradiol IM (Estrogin Farmavet, $\mathrm{SP}$ ). A superovulação ovariana (SO) foi executada pela manhã e à tarde com hormônio folículo estimulante (FSH)(Pluset, Serono, Itália)), administrado diariamente a intervalo de 12 horas entre cada aplicação, durante quatro dias seguidos nos dois grupos, conforme 0 protocolo: dia 6, $75 \mathrm{UI}$; dia 7, $40 \mathrm{UI}$; dia 8, $30 \mathrm{UI}$; dia 9, 20 UI. No dia 8 à tarde administrou-se 500 microgramas de cloprostenol IM (Ciosin - Schering-Plough) e em seguida houve a retirada dos pessários vaginais de todos os animais. Na sequência, foram feitas as observações de cio; os animais foram inseminados duas vezes a intervalos de 12 horas um do outro. Adicionalmente, 0 G1 recebeu junto com a $1^{\underline{a}}$ inseminação artificial (IA), uma dose de 3000 UI de hormônio coriônico gonadotrófico (hCG) (Vetecor, Calier, IV) e o G2 recebeu $1 \mathrm{ml}$ de solução fisiológica, como placebo. Todos os animais tiveram os ovários monitorados mediante ultrassonografia, desde o dia da $1^{\text {a }}$ IA até o dia da coleta dos embriões, mediante aparelho Aloka SSD-550 (Japão), visando a avaliação de folículos, ovulação e corpos lúteos. No $5,5^{\circ}$ dia pós a observação de cio, procedeu-se às lavagens dos cornos uterinos para a colheita dos embriões e avaliação. Concluiu-se que o protocolo utilizado para SO em bubalinos com FSH, foi eficiente, ao promover significativo numero de folículos antrais maiores que $8,0 \mathrm{~mm}$ de diâmetro no dia do cio e que o hCG mostrou-se significativamente eficiente, pois proporcionou maior \% de ovulações no grupo tratado $(p<0,05)$. Não foram detectadas diferenças significativas entre os grupos no tocante ao numero de embriões colhidos.
\end{abstract}

Palavras-chave: Superovulação ovariana, hormônio coriônico gonadotrófico, taxa de ovulação, produção de embriões, búfalas.

ABSTRACT - The aim of the present research work was to optimize ovarian superovulation rate and the embryo production in Murrah and Mediterranean buffaloes around the $60^{\text {th }}$ day after parturition. Fourteen animals were divided in two groups of 7 animals each, $\mathrm{G}_{1}$ (treated animals) and $\mathrm{G}_{2}$ (untreated animals). All animals were subjected to a step of oestrus synchronization by receiving at the 0 day (OD) a vaginal pressary with progestagen (CIDR-B, Intervet), followed by the administration, in the morning of the day 1 (D1), of $3 \mathrm{mg}$ stradiol benzoate IM (Estrogin, Farmavet, SP). The ovarian superovulation (SO) was carried out daily, at intervals of 12 hours in the morning and the afternoon by means of the administration of follicule stimulant hormone (FSH) (Pluset, Serono, Italy) in both groups of experimental animals, during four days according to the following protocol: at the $6^{\text {th }}$ day, $75 \mathrm{IU}$; at the $7^{\text {th }}$ day, 40 $\mathrm{IU}$; at the $8^{\text {th }}$ day, $30 \mathrm{IU}$; and at the $9^{\text {th }}$ day, $20 \mathrm{IU}$. At the afternoon of the $8^{\text {th }}$ day, administration of $500 \mu \mathrm{g}$ of cloprostenol IM (Closin-Schering-Plough) was performed followed by the withdraw of the vaginal pessaries from all animals. Then, observations on the estrus of the experimental animals has been carried out. For this purpose the animals were inseminated twice at intervals of 12 hours. Additionaly, all animals from the G1, together with the first artificial insemination $(\mathrm{Al})$ received a dose of $3000 \mathrm{UI}$ of gonadotrophic chorionic hormone $(\mathrm{GCH})$ (Vetecor, Calier, IV) while the $\mathrm{G} 2$ animals received $1 \mathrm{ml}$ of saline as placebo. All animals from both $\mathrm{G} 1$ and $\mathrm{G} 2$ groups had their ovaries monitored by ultrasonography from the first day of the Al up to the day when they had their embryos collected. Ultrasography has been carried out by means of an Aloka SSD-550 (Japan) instrument aiming to evaluate the follicles, the ovulation, and the corpora lutea. At the $5.5^{\text {th }}$ after the estrus observation a washing of the uterine cornus has been carried out for the embryos harvesting and evaluation. According to the present experiment, it was possible to conclude that the 
protocol used to promote superovulation (SO) in buffaloes by means of FSH showed to be efficient resulting in the production of significant number of antral follicles larger than $8.0 \mathrm{~mm}$ diameter at the estrus day and that the gonadotropohic corionic hormone showed significant efficiency by displaying larger percentual ovulations in the treated group $(p<0.05)$. In regard to the number of embryos collected no significant differences were detected between both the experimental groups.

Key-words: ovary superovulation, chorionic gonadotropin hormone, ovulation rate, embryo production, buffalo.

\section{Introdução}

Segundo a Organização para a Agricultura e Alimento (FAO, 2005), o rebanho bubalino mundial cresceu 7,8\%, aumentando a produção de leite em 28,3 \%.

No Brasil, a bubalinocultura iniciou-se por volta de 1890, com animais importados da Itália e Índia, contabilizando atualmente um rebanho de aproximadamente 3 milhões de cabeças, segundo estimativas da Associação Brasileira de Criadores de Bubalinos (ABCB, 2000). Esta espécie tem sido bastante utilizada nas criações, onde há intensa densidade pluviométrica, como é o caso das regiões litorâneas dos estados do Centro-Sul Brasileiro.

As biotécnicas reprodutivas aplicadas aos bubalinos, tiveram no Brasil, um significativo incremento de pesquisas na última década, impulsionadas principalmente por estudos de BARUSELLI et al. (1999a,b); BARUSELLI et al. (2000a); BARUSELLI et al. (2002); CARVALHO et al. (2002); BARUSELLI e CARVALHO (2005) e CARVALHO et al. (2005).

As primeiras ovulações múltiplas e transferência de embriões (TE) nos bubalinos foram idealizadas em 1983 na Flórida por DROST (1983), registrando o nascimento do primeiro produto obtido com essas técnicas.

Pesquisas encetadas por BARUSELLI e CARVALHO (2005) e por CARVALHO et al. (2005) demonstraram a viabilidade do emprego de dispositivos intravaginais de progesterona $\left(\mathrm{P}_{4}\right)$, associados a promotores de crescimento folicular e a indutores de ovulação na estação reprodutiva desfavorável, possibilitando o emprego da inseminação artificial em tempo fixo (IATF) na espécie bubalina, durante todo o ano, favorecendo a distribuição dos partos e a produção de leite, contornando o período de entressafra, no Centro Sul Brasileiro.

Mais recentemente BARUSELLI et al. (2001) e CARVALHO et al. (2005) realizaram pesquisas empregando o hormônio coriônico gonadotrófico (hCG) e os fatores de liberação de gonadotrofinas $(\mathrm{GnRH})$ como indutores de ovulação em búfalas.

O presente estudo objetivou otimizar a superovulação ovariana, a taxa de ovulação e a produção de embriões em búfalas na região litorânea do estado do Paraná, mediante a utilização de hCG.

\section{Material e Método}

No estudo foram utilizadas búfalas da raça Murrah e Mediterrâneo de propriedade do Instituto Agronômico do Paraná (IAPAR- Morretes,PR). Foram empregadas 14 fêmeas pluríparas em regime semi-estabulado de manejo, peso médio de $470 \mathrm{Kg}$, em torno do $60^{\circ}$ dia pós parto e lactantes. Os animais possuíam o escore da condição corporal em torno de 3,0 a 3,5 na escala de 0 a 5 segundo dados de EDMONDSON et al. (1989). A produção média diária de leite era de 4,0 L/dia. A alimentação dos animais era composta por Brachiaria mutica, Brachiaria radicans, Brachiaria humidicola, Axonopus spp, Paspalum spp., Hemarthria altíssima ao pastoreio; e quando estabuladas as búfalas recebiam Pennisetum purpureum e Saccharum officinarum picadas e levadas ao cocho (cerca de $35 \mathrm{~kg} / \mathrm{cabeça/}$ dia) e $2 \mathrm{~kg} /$ cabeça/dia de milho moído com minerais. Todos os animais eram vermifugados e vacinados.

Os animais foram divididos ao acaso em 2 grupos (G1 = tratado e o G2 não-tratado), sendo cada grupo composto por 7 animais.

Para a sincronização do estro todos os animais receberam no dia 0 (D0 ) um pessário vaginal com progestágeno (CIDR-B, Intervet); no dia 1 (D1) pela manhã foi feita aplicação de $3 \mathrm{mg}$ de benzoato de estradiol IM (Estrogin, Farmavet, SP). A superovulação ovariana (SO) foi executada pela manhã e à tarde com hormônio folículo estimulante (FSH)(Pluset, Serono, Itália)), administrado diariamente a intervalo de 12 horas entre cada aplicação, durante quatro dias seguidos nos dois grupos, conforme o seguinte protocolo: dia 6, 75 UI; dia 7, 40 UI; dia 8, 30 UI; dia 9, 20 UI. No dia 8 à tarde, administrou-se 500 microgramas de cloprostenol (Ciosin - Schering-Plough) e em seguida houve a retirada dos pessários vaginais de todos os animais. A seguir foram feitas as observações de cio; os animais foram inseminados duas vezes a intervalos de 12 horas um do outro. $\mathrm{O} G 1$ recebeu junto à 1a . inseminação artificial (IA), uma dose de 3000 UI de hCG (Vetecor, Calier, IV) e o G2, $1 \mathrm{ml}$ de solução fisiológica, como placebo. Todos os animais tiveram os ovários monitorados mediante ultrassonografia, desde o dia da primeira IA até o dia da coleta dos embriões, por aparelho Aloka SSD-550 (Japão), visando a avaliação de folículos, ovulação e corpos lúteos. No 5,5 dia após a observação de cio, procedeu-se às lavagens dos cornos uterinos, para a colheita dos embriões e avaliação. Os dados obtidos foram submetidos à análise estatística do teste $\mathrm{T}$ de Student e do qui-quadrado segundo CAVALLI -SFORZA (1974).

\section{Resultados e Discussão}

Os trabalhos de pesquisas na área da reprodução, com bubalinos na região do litoral paranaense são escassos. Criadores da espécie estão a exigir melhor 
performance das búfalas, visando principalmente aumento da produção de leite e de animais no geral.

Semelhantemente ao que ocorre com a SO em bovinos (STOCK et al., 1996), BARUSELLI (2000), BARUSELLI e CARVALHO (2005)) observaram que búfalas respondem ao tratamento ovariano superovulatório. Os resultados obtidos no presente estudo (TABELA 1), corroboram relatos de BARUSELLI (2000), ao se verificar 12,6 foliculos com diâmetro maior que $8,0 \mathrm{~mm}$, significando que as búfalas responderam ao tratamento hormonal administrado. Neste estudo, os animais, tanto tratados como os não tratados, demonstraram semelhante resposta concernente ao numero de folículos maiores que $8 \mathrm{~mm}$ de diâmetro.

TABELA 1 - ALGUNS PARÂMETROS REPRODUTIVOS DE BÚFALAS SUPEROVULADAS COM HORMÔNIO FOLÍCULO ESTIMULANTE, INDUZIDAS À OVULAÇÃO COM HORMONIO CORIONICO GONADOTROFICO. COLETA E AVALIAÇÃO DOS EMBRIÕES. MORRETES, PR, 2005. $(n=14)(x \pm s)$.

\begin{tabular}{lll}
\hline & Grupo tratado & Grupo não-tratado \\
\hline Número de animais & 7 & 7 \\
No. de folículos $>8 \mathrm{~mm}$ de $\varnothing$, no estro & $12,7 \pm 6,6^{\mathrm{a}}$ & $12,2 \pm 6,8^{\mathrm{a}}$ \\
No. de ovulações & $9,9 \pm 4,9^{\mathrm{a}}$ & $7,0 \pm 5,0^{\mathrm{b}}$ \\
Taxa de ovulação média (\%) & $78^{\mathrm{a}}$ & $57^{\mathrm{b}}$ \\
№ de corpos lúteos (5,5 dia) após IA & $7,5 \pm 4,3^{\mathrm{a}}$ & $4 \pm 2,5^{\mathrm{b}}$ \\
№ folículos $>8$ mm (5,5 dia) após IA & $2,4 \pm 2,3^{\mathrm{a}}$ & $4,2 \pm 5,1^{\mathrm{b}}$ \\
Média de embriões/animal & $2,4 \pm 1,6^{\mathrm{a}}$ & $1,7 \pm 1,0^{\mathrm{a}}$ \\
\hline
\end{tabular}

Letras iguais na mesma linha $=p>0,05$ (NS);

Letras diferentes na mesma linha $=p<0,05(S)$.

Verifica-se, porém, que nos dados relativos ao número de ovulações, à taxa de ovulação e ao numero de corpos lúteos no dia da colheita dos embriões (TABELA 1 ), comparando-se os dois grupos de animais, houve diferenças $(p<0,05)$ nas três variáveis supracitadas em favor do grupo tratado, evidenciando a eficiência do hCG, ao se tratar de ovulações nesta espécie animal. A taxa de ovulação dos animais tratados (78 \%), foi superior à obtida por BARUSELLI (2000) (62,8\%).

A utilização do hCG demostrou efeitos significativos $(p<0,05)$ no tocante ao menor numero de folículos maiores que $8 \mathrm{~mm}$, existentes no grupo tratado, no dia da colheita dos embriões, confirmando dados de ovulação, superiores no grupo tratado restando 2,4 folículos contra 4,2 no não-tratado (TABELA 1).

Muito embora o grupo tratado com o hCG, tenha proporcionado maior numero de embriões por animal (TABELA 1, p>0,05), em relação ao não-tratado, a quantidade de embriões recuperados foi baixa para ambos os grupos em relação ao numero de ovulações e corpos lúteos detectados via ultrassonografia. Segundo relatos de BARUSELLI (2000) e aqui confirmados, búfalas apresentam dificuldades na recuperação embrionária à lavagem uterina após SO, provavelmente em função do trato reprodutivo dos bubalinos ser de tamanho reduzido (VALE, 1988), podendo haver o comprometimento da captação oocitária, em virtude das dificuldades de orientação espacial das fímbrias do infundíbulo sobre a superfície ovariana, como discutido por SHAW et al. (1995) em bovinos e por BARUSELLI (2000) em bubalinos. Elevadas concentrações de estrógeno em búfalas superovuladas podem comprometer os índices de recuperação embrionária (MISRA et al., 1998), bem como ovulações assincronicas, segundo BARUSELLI (2000).

O uso do hCG mostrou-se eficiente para otimizar as taxas de ovulação e aumentar a formação de estruturas luteínicas, porém seu uso não foi significativo para melhorar o número de embriões recuperados. Outros pesquisadores tais como BARUSELLI et al. (1999b), BARUSELLI et al. (2000b), CARVALHO et al. (2000), obtiveram semelhantes resultados.

\section{Conclusões}

Dos resultados obtidos concluiu-se que:

- o protocolo utilizado para SO em bubalinos com FSH, foi eficiente pois promoveu significativo número de folículos antrais nos ovários dos animais:

- o hCG mostrou-se significativamente eficiente ao promover maior percentual de ovulações no grupo tratado, comparativamente ao não-tratado $(p<0,05)$;

- não foram detectadas diferenças significativas entre os grupos no tocante ao número de embriões produzidos, muito embora o grupo tratado tenha gerado maior número de embriões.

\section{Agradecimentos}

Ao Instituto Agronômico do Paraná - IAPAR - pela possibilidade da execução do trabalho. 


\section{Referências}

ABCB - Associação Brasileira dos Criadores de Búfalos - boletim informativo, agosto de 2000, 12p.

BARUSELLI, P.S. Simpósio sobre controle farmacológico do ciclo estral em ruminantes,. 31 de maio a 2 de junho de 2000, Universidade de São Paulo, São Paulo, p.190-254.

BARUSELLI, P.S.; MADUREIRA, E.H.; VISINTIN, J.A.; PORTO-FILHO, R.; CARVALHO, N.A.T.; CAMPANILE, G.; ZICARELLI, L. Failure of oocyte entry into oviduct in superovulated buffalo. Theriogenology, v.53, p.491, 2000a.

BARUSELLI, P.S.; MADUREIRA, E.N.; BRAGA, D.P.A. F.; PORTO-FILHO, R.; CARVALHO, N. A. T.; FRANZOLIN, R. Efeito da administração de $\mathrm{GnRH}$ na taxa de ovulação em búfalas superovuladas. Revista Brasileira de Reprodução Animal, v. 25, p. 549-52, 2001.

BARUSELLI, P.S.; CARVALHO, N.A.T.; HENRIQUEZ, C.H.P.; AMARAL, R.; NICHI, M. Synchronization of ovulation for timed artificial insemination during the off breeding season in the buffalo. In: Buffalo Symposium of Americas, 1, 2002, Belém. Proceedings ... Belém: [s.n.], 2002. p.418-420.

BARUSELLI, P.S.; MUCCIOLO, R.; ARRUDA, E.H.; MADUREIRA, E.H.; AMARAL R.; ASSUMPÇÃO, M.E. O. A.. Embryo recovery rate in superovulated buffalo. Theriogenology, v. 51, p. 401, 1999a.

BARUSELLI, P.S.; CARVALHO, N.A.T. Biotecnologias da reprodução em bubalinos (Bubalus bubalis). Revista Brasileira de Reprodução Animal, v.29,n.1,p.4-17, 2005.

BARUSELLI, P.S.; MADUREIRA, E.H.; BARNABE, R.C.; BERBER, R.C.A.; AMARAL, R. Timed insemination using synchronization of ovulation in buffalo. In International Congress on Animal Reproduction, $14^{\text {th }}, 2000$, Stockholm: abstracts... Stockholm: ICAR, v.2, p. 14-18, 2000b.

BARUSELLI, P.S.; MADUREIRA, E.H.; VISINTIN, J.A.; BARNABE, V.H.; BARNABE, R.C.; AMARAL, R. Inseminação artificial em tempo fixo com sincronização da ovulação em bubalinos. Revista Brasileira de Reprodução Animal, v.23,p.360-362, 1999b.

CARVALHO, N. A. T.; BARUSELLI, P. S.; VISINTIN, J. A.; MADUREIRA, E. H.; ZICARELLI, L.; D' OCCHIO, M.J. Control of ovulation with a $\mathrm{GnRH}$ agonist after superestimulation of follicular growth in buffalo: fertilization and embryo recovery. Theriogenology, v.58, p.1641-1650, 2002.
CARVALHO, N.A.T.; BARUSELLI, P.S.; ZICARELLI, L.; MADUREIRA, E.H.; VISINTIN, J.A.; D'OCCHIO, M.J. Use of a $\mathrm{GnRH}$ agonist to control ovulation subsequent to superstimulation of follicular growth in buffalo. Theriogenology, v. 53, p. 496, 2000.

CARVALHO, N.A.T.; CARVALHO, M.V.; VISINTIN, J.A.; VANNUCCI, F.S.; SÁ FILHO, M.F.; NICHI, M.; REICHERT, R.H.; BARUSELLI, P.S. Uso de dispositivos intravaginais de progesterona associados ao hCG ou GnRH para sincronização da ovulação em búfalas na estação reprodutiva desfavorável. In: Congresso Brasileiro de Reprodução Animal, 16, 2005, Goiânia, 2005. Anais ... Belo Horizonte: CBRA, 2005. (CD-ROM).

CAVALLI-SFORZA,L. Biometrie - Grundzuge biologisch-medizinischer statistic. Stuttgart, Gustav Fischer Verlag, 1974, 212 p.

DROST, M. Embryo transfer in water buffalo (Bubalus bubalis). Theriogenology, v.20 p573-84, 1983.

EDMONDSON, A.J.; LEAN, I.J.; WEAVER, L.D. A body condition scoring chart for Holstein cows. Journal of Dairy Science, v.72, p.68-78,1989.

FOOD AND AGRICULTURE ORGANIZATION - FAO,. FAOSTAT: Agriculture data. Disponivel em:Http:// $\mathrm{f} a$ o s t a t. f a o. org/f a o s t a t / collections? versio=ext\&hasbulk=0\&subset=Agriculture. Acesso em 30/04/2005.

MISRA, A.K.; KASIRAJ, R.; MUTHA, R.M.; RANGAREDDY, N.S.; JAISWAL, R.S.; PANT, H.C. Rate of transport and development of preimplantation embryo in the superovulated buffalo (Bubalus bubalis). Theriogenology, v.50,p.637$649,1998$.

SHAW, D.W.; FARIN, P.W.; WASHBURN, S.P.; BRITT, J.H. Effect of retinol palmitate on ovulation rate and embryo quality in superovulated cattle. Theriogenology, v.44,p.51-58,1995.

STOCK, A.E.; ELLINGTON, J.E.; FORTUNE,J .E. A dominant follicle does not affect follicular recruitment by superovulatory doses of FSH in cattle but can inhibit ovulation. Theriogenology, v.45,p.1091-1102, 1996.

VALE, W.G. Bubalinos: fisiologia e patologia da reprodução. Campinas, Fundação Cargil, 1988,86 p.

Recebido para publicação:

$02 / 02 / 2006$ Aprovado: $14 / 06 / 2006$ 